\title{
Optimal Loading and Protection of Multi-state Systems Considering Performance Sharing Mechanism
}

\author{
Hui Xiao, Daimin Shi, Yi Ding and Rui Peng
}

\begin{abstract}
Engineering systems are designed to carry the load. The performance of the system largely depends on how much load it carries. On the other hand, the failure rate of the system is strongly affected by its load. Besides internal failures, such as fatigue and aging process, systems may also fail due to external impacts such as nature disasters and terrorism. In this paper, we integrate the effect of loading and protection of external impacts on multi-state systems with performance sharing mechanism. The objective of this research is to determine how to balance the load and protection on system elements. An availability evaluation algorithm of the proposed system is suggested and the corresponding optimization problem is solved utilizing genetic algorithms.
\end{abstract}

Index Terms - multi-state systems, performance sharing, load dependent failure, protection, universal generating function, genetic algorithms

\section{NOMENCLATURE}

$e_{i}: \quad$ multi-state element $i$

$E_{j}: \quad$ the set of multi-state elements located at sub-system $j$

$L_{i}: \quad$ the load on $e_{i}$

$g\left(L_{i}\right)$ : the performance of $e_{i}$ when its load is $L_{i}$

$L_{i, \min }$ : the minimum allowable load on $e_{i}$

$L_{i, \max }:$ the maximum allowable load on $e_{i}$

$N: \quad$ the number of sub-systems connected in series

$M: \quad$ the total number of multi-state elements

$G_{i}: \quad$ the random performance of $e_{i}$

$O_{j}: \quad$ the random performance of sub-system $j$

$W_{j}$ : the random demand of sub-system $j$

$C: \quad$ the random transmission capacity of the common bus

This research is partially supported by the China NSFC under Grant 71231001, 11001005 and 71301009, the Fundamental Research Funds for the Central Universities under FRF-TP-14-051A2 and by the MOE PhD Supervisor Fund 20120006110025.

Hui Xiao is with School of Statistics, Southwestern University of Finance and Economics, Chengdu, China (e-mail: msxh@swufe.educ.cn).

Daimin Shi is with School of Statistics, Southwestern University of Finance and Economics, Chengdu, China (e-mail: shidm@swufe.educ.cn).

Yi Ding is with College of Electrical Engineering, Zhejiang University, Zhejiang, China(e-mail: yding@elektro.dtu.dk).

Rui Peng is with Dongling School of Economics and Management, University of Science and Technology Beijing, Beijing, China (email:pengrui1988@ustb.educ.cn). performance sharing system

$S_{j}$ : the random performance surplus of sub-system $j$

$D_{j}: \quad$ the random performance deficiency of sub-system $j$

$Z$ : the random amount of redistributed performance

$\hat{D}: \quad$ the system deficiency after redistribution

$x_{i}: \quad$ the protection effort allocated to $e_{i}$

$f: \quad$ constant frequency of external impacts

$d$ : $\quad$ expected impacts intensity

$c_{r i}: \quad$ constant repair cost of $e_{i}$ for internal failures

$c_{p_{2} i}: \quad$ constant repair cost of $e_{i}$ for failure caused by external impacts

$c_{p_{1} i}: \quad$ unit protection cost of $e_{i}$

$t_{r i}$ : constant repair time of $e_{i}$ for internal failures

$t_{p i}$ : constant repair time of $e_{i}$ for failure caused by external impacts

$T: \quad$ system lifetime

$C_{T}: \quad$ the total system cost

$A^{*}: \quad$ the pre-specified system availability requirement

$\theta(j)$ : the number of states of the demand of sub-system $j$

$q_{j r}$ : the probability that the demand of sub-system $j$ is in state $r$

$w_{j r}$ : the demand of sub-system $j$ when it is in state $r$

$Q$ : $\quad$ The number of states of the common bus performance sharing system

$\alpha_{\beta}$ : the probability that the common bus performance sharing system is in state $\beta$

$\varsigma_{\beta}$ : the transmission capacity when the common bus performance sharing system in state $\beta$

\section{INTRODUCTION}

A multi-state series-parallel system consists of $N$ sub-systems connected in series and each sub-system contains several multi-state elements connected in parallel [1-6]. The system fails if and only if at least one of the sub-systems cannot meet its required demand. In the literature, most of the works assume that each sub-system satisfies its own demand individually. However, many examples in real situations such as power systems, communication systems and data processing systems indicate that the surplus performance from one 
sub-system can be transmitted to other sub-systems that are experiencing the performance deficiency. This type of performance sharing mechanism was first studied in [7], and extended in $[8,9]$.

Many research works have considered the element allocation and maintenance of the multi-state series-parallel system $[5,10$, 11 ], but very limited work considered the effect of loading on the system elements. However, a majority of the engineering systems are designed to carry the load, such as coal conveyors, cargo trucks, and power generating units. The performance of such systems depends on the amount of load it is carrying. Besides, many studies have empirically shown that the failure rate of the system element is strongly affected by its working load [12, 13]. Therefore, it is important to consider the effect of loading when analyzing the availability of multi-state systems.As a result, several recent research works have studied the optimal loading of multi-state systems [14-19].

Besides the internal failures such as aging process and fatigue, the system element may also fail due to external impacts such as natural disasters and terrorism [20]. One approach for improving the survivability of system elements is to make defensive investment to protect the elements [21-23]. The probability that an element is destroyed by the external impact is usually modeled as a function of the external impacts intensity and the protection effort allocated on the element [24-27]. The survival probability of an element is higher if more protection effort is allocated onto it. The optimal trade-off between investment into the maintenance and protection of the elements in a simple parallel system that is subject to both internal failure and natural disasterswas studied in [27].

In this paper, we consider a multi-state series-parallel system with common bus performance sharing as shown in Figure 1. The performance of each sub-system is the cumulative performance of the elements within the sub-system. If the performance of any sub-system is more than its demand, the surplus performance can be transmitted to any other deficient sub-system via the common bus. However, the total amount of the performance that can be transmitted among different sub-systems is subjected to the capacity of the common bus. In this research, the capacity is also assumed to be a random variable since the common bus may be concurrently used by several systems.The performance of any system element depends on the load it is carrying. In general, the working performance of each element increases with increasing load. However, increasing the load will also increase the failure rate of the element, and thus reduce the availability of the element. Therefore, the expected performance of the element is not a monotonic function of its load. Besides internal failures, the system elements are also subject to external impacts that occur with a constant frequency. Hence, it is important to consider providing protection to the system elements to increase their survivability against external impacts. For example, if the external impacts are from earthquakes, anti-seismic devices can be installed to protect system elements.

Comparing with other works on common bus systems, we have considered both the effects of internal failure and external impacts on the system elements. To increase the system availability, the system elements are protected and immediately repaired in case of failure. Besides, the effect of load on element failure rate is considered. Since increasing load can increase the element failure rate, but also increases the element performance, the relationship between load and the system availability is already non-monotonic. With the incorporation of the external impact, it is impossible to give an intuitive answer to how to load and protect the system element. Therefore, it is essential to propose a framework to solve the joint optimization problem of system loading and protection.

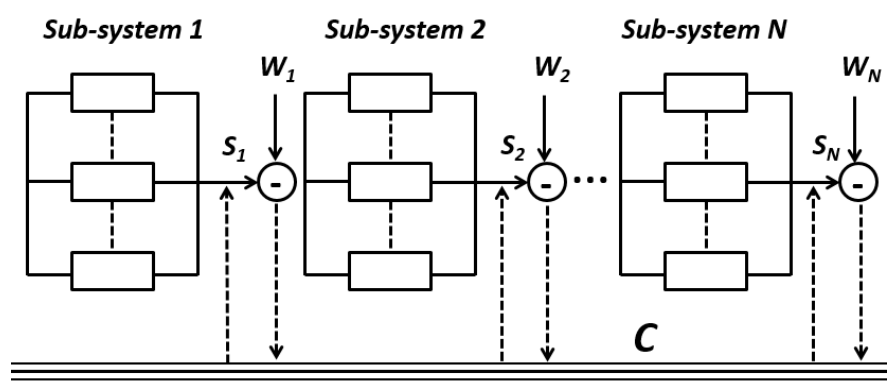

Fig.1. Series-parallel system with common bus performance sharing

The rest of the paper is organized as follows. Section 2 discusses the load dependent failure rate and performance. Section 3 provides the availability modeling of the multi-state series-parallel system with performance sharing. Section 4 formulates the joint optimization model of loading and protection. The availability evaluation algorithm of the proposed system based on universal generating function is suggested in Section 5. Section 6 proposes the optimization technique. Numerical experiments are provided in Section 7. The paper is concluded in Section 8.

\section{LOAD DEPEndent FAILURE RATE AND PERFormance}

Every multi-state element $e_{i}$ considered in this paper is able to carry load of different values. However, element $e_{i}$ can only be in two states: failure state with zero performance and functioning state with working performance $g_{i}\left(L_{i}\right)$, where $L_{i}$ is the load carried by element $e_{i}$ and $g_{i}\left(L_{i}\right)$ which denotes the performance of element $e_{i}$ is a function of load $L_{i}$. The load on element $e_{i}$ can vary from $L_{i, \min }$ to $L_{i, \max }$, where $L_{i, \min }$ and $L_{i, \max }$ denote the minimum and maximum allowable load on element $e_{i}$ respectively. This is the reason why element $e_{i}$ is considered to be multi-state. As discussed earlier, the expected performance of element $e_{i}$ is not a monotonic function of the load $L_{i}$.

It can be difficult to distinguish the load and the performance in some situations since they can be considered the same. For example, the pressure on a pipe which carries fluid in a laminar flow mode is proportional to the volume of the flow, i.e., the throughput (performance) is proportional to the pressure (load). However, the load-performance relationship can be nonlinear. For instance, consider a pipe carrying fluid in a turbulent flow 
mode. The pressure on the pipe is a non-linear function of volume of the flow.

The load dependent performance function $g_{i}\left(L_{i}\right)$ can take different expressions depending on the situations. Without loss of generality, the function measuring relationship between the performance and the load can be assumed as follows.

$$
g_{i}\left(L_{i}\right)=a_{i}+c_{i} L_{i}
$$

where $a_{i}$ and $c_{i}$ are the coefficients of the linear equation.

To analyze the availability of systems made up by elements with load dependent failure rates, the load failure relationship must be known beforehand since the availability of each element can be derived based on its failure rate. In the literature, the accelerated life test models play an important role in determining the load-failure rate relationships. The existing accelerated life test models are summarized in [28]. In this paper, the commonly used proportional hazard model (PHM) will be discussed and used in the numerical experiments.

PHM, which was first proposed in [29], has received popularity in the field of reliability engineering in recent years [30-32].PHM states that the failure rate of an element is the product of the baseline hazard rate and factors based on the conditions. In general, PHM can be expressed as follows.

$$
h(t \mid \boldsymbol{X})=h_{0}(t) \cdot e^{\left(\beta_{1} X_{1}+\beta_{2} X_{2}+\cdots+\beta_{d} X_{d}\right)}
$$

where $h_{0}(t)$ denotes the baseline hazard rate as a function of time. $X_{1}, \cdots, X_{d}$ are the factors that affect the hazard rate function and $\beta_{1}, \cdots, \beta_{d}$ are the corresponding coefficients.

This paper assumes that load is the only factor that affects the failure rate of each element. Thus, we can simplify the PHM to be a single factor model.

$$
h(t \mid L)=h_{0}(t) \cdot e^{L \beta}(3)
$$

In this research, we consider the scenario where the load on each element is static, i.e., the load on each element does not change during the entire system lifetime.In addition, we assume that the baseline failure rate is constant across time. Given the above conditions, equation (2) can be further simplified to be the following expression.

$$
h(L)=h(t \mid L)=\lambda \cdot e^{L \beta}(4)
$$

where $\lambda=h_{0}(t)$ for all $t$ is the baseline failure rate.

PHM is the most frequently used accelerated life test model in reliability, though there exists many other models in the literatures. Different models take different expressions, but they can be similarly applied to the considered problem like PHM.

\section{AVAILABILITY MODELING}

The multi-state series-parallel system considered in this paper consists of $N$ sub-systems connected in series. In sub-system $j$, there are $H_{j}$ multi-state elements connected in parallel. In total, there are $M$ multi-state elements within the system, i.e., $\sum_{j=1}^{N} H_{j}=M$. Let $W_{j}$ denote the demand of sub-system $j$. The demand is a random variable with probability mass function $q_{j, r}=P\left(W_{j}=w_{j, r}\right)$ and $\sum_{r=1}^{\theta(j)} q_{j, r}=1$, where $q_{j, r}$ denotes the probability that the demand of sub-system $j$ is in state $r$ with the rate of demand being $w_{j, r}$. In total, there are $\theta(j)$ possible states for the random demand $W_{j}$. The performance of sub-system $j$ is the cumulative performance of all elements connected in parallel. Let $G_{i}$ denote the performance of element $e_{i}$. Given that the load on element $e_{i}$ is $L_{i}$, it can be known that $G_{i}=g_{i}\left(L_{i}\right)$ if element $e_{i}$ is working and $G_{i}=0$ if element $e_{i}$ fails. Therefore, the total performance of sub-system $j$ can be denoted as follows.

$$
O_{j}=\sum_{e_{i} \in E_{j}} G_{i}
$$

where $E_{j}$ denotes the set of elements within sub-system $j$.

Hence, the performance surplus of the series-parallel system with common bus performance sharing can be evaluated by summing up performance surplus from each sub-system.

$$
S=\sum_{j=1}^{N} S_{j}=\sum_{j=1}^{N} \max \left(O_{j}-W_{j}, 0\right)(6)
$$

Similarly, the performance deficiency of the system can be expressed as follows.

$$
D=\sum_{j=1}^{N} D_{j}=\sum_{j=1}^{N} \max \left(W_{j}-O_{j}, 0\right)(7)
$$

The total amount of transmission among different sub-systems cannot be greater than the minimum of the surplus and deficiency. In addition, the total amount of transmission is further constrained by the capacity of the performance sharing system. The capacity of the performance sharing system is a random variable $C$ with the probability mass function: $\alpha_{\beta}=P\left(C=\varsigma_{\beta}\right)$ and $\sum_{\beta=1}^{Q} \alpha_{\beta}=1$. Therefore, the total amount of transmission is the minimum of $S, D, C$.

$$
\begin{aligned}
& Z=\min (S, D, C) \\
& =\min \left(\sum_{j=1}^{N} \max \left(O_{j}-W_{j}, 0\right), \sum_{j=1}^{N} \max \left(W_{j}-O_{j}, 0\right), C\right)^{(8)}
\end{aligned}
$$


The system performance deficiency after performance redistribution is,

$$
\begin{aligned}
& \hat{D}=D-Z=D-\min (S, D, C)=\max (0, D-\min (S, C)) \\
& =\max \left(0, \sum_{j=1}^{N} \max \left(W_{j}-O_{j}, 0\right)-\min \left(\sum_{j=1}^{N} \max \left(O_{j}-W_{j}, 0\right), C\right)\right)
\end{aligned}
$$

The availability of the system is the fraction of time when no performance deficiency exists in the system. Mathematically, it can be denoted as follows.

$$
\begin{aligned}
& R=\mathrm{P}\{\hat{D}=0\} \\
& =\mathrm{P}\left\{\max \left(0, \sum_{j=1}^{N} \max \left(W_{j}-O_{j}, 0\right)-\min \left(\sum_{j=1}^{N} \max \left(O_{j}-W_{j}, 0\right), C\right)\right)=0\right\}
\end{aligned}
$$

It can be verified that the proposed series-parallel system with performance sharing mechanism reduces to a general series-parallel system if $C=0$ or a parallel system with all elements connected in parallel if $C=\infty$.

\section{OPtimal LOADING AND PROTECTION MODEL}

Each element within the series-parallel system can be arbitrarily loaded subjected to the minimum and maximum allowable limit, i.e., the load $L_{i}$ on element $e_{i}$ must satisfy the condition $L_{i, \min } \leq L_{i} \leq L_{i, \max }$. Given that the load on element $e_{i}$ is $L_{i}$, its failure rate can be derived based on PHM.

$$
h_{i}\left(L_{i}\right)=\lambda_{i} \cdot e^{\beta_{i} L_{i}}
$$

When element $e_{i}$ fails, it will be repaired immediately and the repair is assumed to be perfect. The cost and time needed for repair are $c_{r i}$ and $t_{r i}$ respectively. It is reasonable to assume that the cost and time for repair is constant for the same element.

In addition, the element is also subjected to external impacts. Let $x_{i}$ denote the protection effort allocated to element $e_{i}$ and $c_{p_{1} i}$ be the unit protection cost for element $e_{i}$. The constant frequency of the external impacts is $f$ with the expected impact intensity being $d$. The element vulnerability, which is defined as the conditional probability of an element failure caused by an external impacts, is commonly evaluated using the contest function model as follows [33, 34].

$$
v\left(x_{i}, d\right)=\frac{d^{m}}{x_{i}^{m}+d^{m}}
$$

where $m$ is the contest intensity parameter.
Let $c_{p_{2} i}$ be the constant repair cost for element $e_{i}$ if the failure is caused by external impacts and $t_{p i}$ be the constant repair time for element $e_{i}$. Given that the system lifetime is $T$, the availability of the element $e_{i}$ can be expressed as follows.

$$
\begin{aligned}
A_{i}\left(L_{i}, x_{i}\right) & =\left(T-t_{r i} \cdot \lambda_{i} e^{\beta_{i} L_{i}} \cdot T-f \cdot v\left(x_{i}, d\right) \cdot t_{p i} \cdot T\right) / T \\
& =1-t_{r i} \cdot \lambda_{i} e^{\beta_{i} L_{i}}-f \cdot v\left(x_{i}, d\right) \cdot t_{p i}
\end{aligned}
$$

The total cost includes the repair cost of both internal failures and external impacts and the cost spent on protection. Assuming that the repair costs $c_{r i}$ and $c_{p_{2} i}$ for element $e_{i}$ are constant and independent of the system state, the total system cost can be expressed as follows.

$$
C_{T}=\sum_{i=1}^{M}\left(c_{p_{1} i} \cdot x_{i}+c_{p_{2} i} \cdot f \cdot v\left(x_{i}, d\right) \cdot T+c_{r i} \cdot \lambda_{i} e^{\beta_{i} L_{i}} \cdot T\right)
$$

The first term in the parentheses denotes the investment cost of protecting element $e_{i}$. The second term denotes the repair cost of failures caused by external impacts for element $e_{i}$. The last term represents the repair cost of internal failures for element $e_{i}$.

The objective of this research is to find the optimal loading and protection on each element in order to minimize the total system cost while meeting the pre-specified system availability requirement. Let $\boldsymbol{L}=\left(L_{1}, \cdots, L_{M}\right)$ be the vector representing the load on each element and $\boldsymbol{X}=\left(x_{1}, \cdots, x_{M}\right)$ be the vector denoting the protection effort allocated to each element. Let $A(\boldsymbol{L}, \boldsymbol{X})$ denote the availability of the system given the load on each element is $\boldsymbol{L}$ and protection effort for each element is $\boldsymbol{X}$. $A^{*}$ is the pre-specified system availability requirement. The optimization model can be written as follows.

$$
\begin{array}{ll}
\min & C_{T}=\sum_{i=1}^{M}\left(c_{p_{1} i} \cdot x_{i}+c_{r i} \cdot \lambda_{i} \cdot e^{\beta_{i} L_{i}} \cdot T+c_{p_{2} i} \cdot f \cdot v\left(x_{i}, d\right) \cdot T\right) \\
\text { s.t. } & A(\boldsymbol{L}, \boldsymbol{X}) \geq A^{*} \\
& x_{i} \geq 0, \forall i=1, \cdots, M \\
& L_{i, \min } \leq L_{i} \leq L_{i, \max }, \forall i=1, \cdots, M
\end{array}
$$

In the optimization model above, the objective is to minimize the total system cost $C_{T}$. The decision variables are the load given to each element, i.e., $\boldsymbol{L}=\left(L_{1}, \cdots, L_{M}\right)$, and the protection allocated to each element, i.e., $\boldsymbol{X}=\left(x_{1}, \cdots, x_{M}\right)$. The first constraint requires that the availability of the system for any combination of $\boldsymbol{L}=\left(L_{1}, \cdots, L_{M}\right)$ and $\boldsymbol{X}=\left(x_{1}, \cdots, x_{M}\right)$ must be at least $A^{*}$. The second constraint forces the protection effort of any element to be nonnegative. The last constraint defined the maximum and minimum allowable load on each element. 


\section{Availability Evaluation Algorithm}

To solve the optimization model (15), the availability of the considered system must be evaluated first. In other words, we must find a procedure which can compute the system availability given the the load on each element is $\boldsymbol{L}$ and the protection efforts on each element is $\boldsymbol{X}$.

In this section, we suggest an availability evaluation algorithm for the proposed system based on the universal generating function (UGF) which was first introduced by Ushakov[35]. The UGF technique has been proven to be effective in analyzing the system reliability of different structures such as the networks reliability model [36, 37], sliding window systems[38-40], voting systems [41], linear consecutively connected systems [42], series-parallel systems $[5,9]$. The technique has also been applied to analyze the reliability of various real industrial and engineering systems such as distributed generation systems [43], the stress-strength interference model [44] and maritime transportation systems [45]. A comprehensive summary of the applications of the UGF technique to reliability analysis and optimization can be found in [46].

The UGF for a discrete random variable $X$ is defined as the following polynomial.

$$
u(z)=\sum_{k=1}^{K} \varsigma_{k} \cdot z^{X_{k}}
$$

where the variable $X$ has $K$ possible realizations and $\varsigma_{k}=P\left(X=X_{k}\right)$.

In this paper, element $e_{i}$ is able to support load from $L_{i, \min }$ to $L_{i, \text { max }}$. When the load on element $e_{i}$ is $L_{i}$, the performance of $e_{i}$ is either $g\left(L_{i}\right)$ if it is working or 0 if it fails. Therefore, the UGF of element $e_{i}$ can be written as follows.

$$
u_{i}\left(z \mid L_{i}\right)=\left(1-A_{i}\left(L_{i}\right)\right) \cdot z^{0}+A_{i}\left(L_{i}\right) \cdot z^{g_{i}\left(L_{i}\right)}
$$

where $A_{i}\left(L_{i}\right)$ is the probability that element $e_{i}$ is working given the load on $e_{i}$ is $L_{i}$. Equation (17) indicates that the state distribution of the element $e_{i}$ depends on the load it is carrying.

Consider a pair of elements $e_{e}$ and $e_{f}$ connected in parallel. The total performance of element $e_{e}$ and $e_{f}$ is the cummulative performance of the two elements. Define an operator $\oplus$ to derive the combined UGF of the two elments as follows.

$$
\begin{aligned}
& \oplus\left(u_{e}\left(z \mid L_{e}\right), u_{f}\left(z \mid L_{f}\right)\right) \\
& =\oplus\left(\begin{array}{l}
\left(1-A_{e}\left(L_{e}\right)\right) \cdot z^{0}+A_{e}\left(L_{e}\right) \cdot z^{g_{e}\left(L_{e}\right)}, \\
\left(1-A_{f}\left(L_{f}\right)\right) \cdot z^{0}+A_{f}\left(L_{f}\right) \cdot z^{g_{f}\left(L_{f}\right)}
\end{array}\right) \\
& =\sum_{h_{e}=0}^{1} \sum_{h_{f}=0}^{1}\left(\begin{array}{l}
\left(A_{e}\left(L_{e}\right)\right)^{h_{e}} \cdot\left(1-A_{e}\left(L_{e}\right)\right)^{1-h_{e}} \\
\cdot\left(A_{f}\left(L_{f}\right)\right)^{h_{f}} \cdot\left(1-A_{f}\left(L_{f}\right)\right)^{1-h_{f}}
\end{array}\right) \cdot z^{h_{e} g_{e}\left(L_{e}\right)+h_{f} g_{f}\left(L_{f}\right)}
\end{aligned}
$$

It can be verified that the operator $\oplus$ satisfies the communicative law.

$$
\oplus\left(u_{e}\left(z \mid L_{e}\right), u_{f}\left(z \mid L_{f}\right)\right)=\oplus\left(u_{f}\left(z \mid L_{f}\right), u_{e}\left(z \mid L_{e}\right)\right)
$$

and the associative law,

$$
\begin{aligned}
& \oplus\left(u_{e}\left(z \mid L_{e}\right), u_{f}\left(z \mid L_{f}\right), u_{g}\left(z \mid L_{g}\right)\right) \\
& =\oplus\left(\oplus\left(u_{e}\left(z \mid L_{e}\right), u_{f}\left(z \mid L_{f}\right)\right), u_{g}\left(z \mid L_{g}\right)\right)(20) \\
& =\oplus\left(u_{e}\left(z \mid L_{e}\right) \oplus\left(u_{f}\left(z \mid L_{f}\right), u_{g}\left(z \mid L_{g}\right)\right)\right)
\end{aligned}
$$

Let $E_{j}=\left\{e_{j_{1}}, \cdots, e_{j_{\left|E_{j}\right|}}\right\}$ denote the set of elements connected in parallel within sub-system $j$. The performance of sub-system $j$ is the cumulative performance of elements $e_{j_{1}}, \cdots, e_{{\dot{\left|E_{E}\right|}}_{1}}$. Therefore, the UGF denoting the performance of sub-system $j$ can be derived by applying the operator $\oplus$ over all elements within sub-system $j$.

$U_{j}(z \mid \boldsymbol{L})=\oplus\left(u_{j_{1}}\left(z \mid L_{j_{1}}\right), \cdots, u_{j_{E_{j} \mid} \mid}\left(z \mid L_{j_{E_{j} j} \mid}\right)\right)$

$=\sum_{h_{j_{1}}=0}^{1} \cdots \sum_{h_{j_{E_{j}} \mid} \mid}^{1}\left(\prod_{i=1}^{\left|E_{j}\right|}\left(\left(A_{j_{i}}\left(L_{j_{i}}\right)\right)^{h_{j_{i}}}\left(1-A_{j_{i}}\left(L_{j_{i}}\right)\right)^{1-h_{j_{i}}}\right)\right) \cdot z^{\sum_{i=1}^{\left|E_{i=1}\right|} h_{j_{i}} \cdot g_{j_{i}}\left(L_{j_{i}}\right)}$

Equation (21) expresses the performance distribution of sub-system $j$ using UGF. Similarly, the demand of sub-system $j$ can be expressed as follows.

$$
W_{j}(z)=\sum_{r=1}^{\theta(j)} q_{j r} \cdot z^{w_{j r}}
$$

The combination of the performance rate and demand level of sub-system $j$ constructs a state of sub-system $j$. The state of sub-system $j$ is described by the performance surplus $S_{j}$ and performance deficiency $D_{j}$. Since $S_{j}$ and $D_{j}$ are statistically dependent, a single UGF $\Delta_{j}(z \mid \boldsymbol{L})$ should be derived to represent the state distribution of the sub-system $j$ 
considering both $S_{j}$ and $D_{j} \cdot \Delta_{j}(z \mid \boldsymbol{L})$ can be derived using the following operator $\underset{\oplus}{\oplus}$ as follows.

$$
\begin{aligned}
& \Delta_{j}(z \mid \boldsymbol{L})=U_{j}(z \mid \boldsymbol{L}) \stackrel{\oplus}{\rightarrow} W_{j}(z) \\
= & \left(\sum_{h_{j_{1}}=0}^{1} \cdots \sum_{h_{j_{\mid}} \mid}^{1} \prod_{i=1}^{\left|E_{j}\right|}\left(\left(A_{j_{i}}\left(L_{j_{i}}\right)\right)^{h_{j_{i}}}\left(1-A_{j_{i}}\left(L_{j_{i}}\right)\right)^{1-h_{j_{i}}}\right) \cdot z^{\sum_{i=1}^{\left|E_{j}\right|} h_{j_{i}} g_{j_{i}}\left(L_{j_{i}}\right)}\right) \\
& \stackrel{\oplus}{\rightarrow}\left(\sum_{r=1}^{\theta(j)} q_{j r} \cdot z^{w_{j r}}\right) \\
= & \sum_{b_{j}=1}^{B_{j}} \pi_{b_{j}}(\boldsymbol{L}) \cdot z^{\max \left\{\sum_{i=1}^{\left|E_{j}\right|} h_{j_{i}} g_{j_{i}}\left(L_{j_{i}}\right)-w_{j r}, 0\right\}, \max \left\{w_{j r}-\sum_{i=1}^{\left|E_{j=1}\right|} h_{j_{i}} g_{j_{i}}\left(L_{j_{i}}\right), 0\right\}} \\
= & \sum_{b_{j}=1}^{B_{j}} \pi_{b_{j}}(\boldsymbol{L}) \cdot z^{s_{b_{j}}(\boldsymbol{L}), d_{b_{j}}(\boldsymbol{L})}
\end{aligned}
$$

where $\pi_{b_{j}}(\boldsymbol{L})=\mathrm{P}\left\{\left(S_{j}=s_{b_{j}}(\boldsymbol{L})\right) \cap\left(D_{j}=d_{b_{j}}(\boldsymbol{L})\right)\right\}$.

Define an operator $\otimes$ to compute the UGF that denotes the joint states of sub-system $i$ and sub-system $j$ as follows.

$$
\begin{aligned}
& \Delta_{j}(z \mid \boldsymbol{L}) \otimes \Delta_{i}(z \mid \boldsymbol{L}) \\
& =\left(\sum_{b_{j}}^{B_{j}} \pi_{b_{j}}(\boldsymbol{L}) \cdot z^{s_{b_{j}}(\boldsymbol{L}), d_{b_{j}}(\boldsymbol{L})}\right) \otimes\left(\sum_{b_{i}}^{B_{i}} \pi_{b_{i}}(\boldsymbol{L}) \cdot z^{s_{b_{i}}(\boldsymbol{L}), d_{b_{i}}(\boldsymbol{L})}\right) \\
& =\sum_{b_{j}=1}^{B_{j}} \sum_{b_{i}=1}^{B_{i}} \pi_{b_{j}}(\boldsymbol{L}) \cdot \pi_{b_{i}}(\boldsymbol{L}) \cdot z^{s_{b_{j}}(\boldsymbol{L})+s_{b_{i}}(\boldsymbol{L}), d_{b_{j}}(\boldsymbol{L})+d_{b_{i}}(\boldsymbol{L})}
\end{aligned}
$$

It can be proven that $\otimes$ satisfies the communicative law and associative law like operator $\oplus$. Hence, the UGF of the total system performance surplus and deficiency can be obtained by applying the operator $\otimes$ over all sub-systems.

$$
\begin{aligned}
& \Gamma(z \mid \boldsymbol{L})=\otimes\left(\Delta_{1}(z \mid \boldsymbol{L}), \cdots, \Delta_{N}(z \mid \boldsymbol{L})\right) \\
& =\otimes\left(\left(\sum_{b_{1}}^{B_{1}} \pi_{b_{1}}(\boldsymbol{L}) \cdot z^{s_{b_{1}}(\boldsymbol{L}), d_{b_{1}}(\boldsymbol{L})}\right), \cdots,\left(\sum_{b_{N}}^{B_{N}} \pi_{b_{N}}(\boldsymbol{L}) \cdot z^{s_{b_{N}}(\boldsymbol{L}), d_{b_{N}}(\boldsymbol{L})}\right)\right) \\
& =\sum_{b}^{B} \pi_{b}(\boldsymbol{L}) \cdot z^{s_{b}(\boldsymbol{L}), d_{b}(\boldsymbol{L})}
\end{aligned}
$$

where $\pi_{b}(\boldsymbol{L})=\mathrm{P}\left\{\left(S=s_{b}(\boldsymbol{L})\right) \cap\left(D=d_{b}(\boldsymbol{L})\right)\right\}$.

Equation (25) represents the UGF for the total system performance surplus and deficiency. However, the capacity of the performance sharing system has not been considered yet. Recall that the transmission capacity of the common bus performance sharing system is a random variable $C$ with the probability mass function: $\alpha_{\beta}=P\left(C=\varsigma_{\beta}\right)$ and $\sum_{\beta=1}^{Q} \alpha_{\beta}=1$. We can express the state distribution of the random variable $C$ using UGF by the following polynomial.

$$
\eta(z)=\sum_{\beta=1}^{Q} \alpha_{\beta} \cdot z^{\varsigma_{\beta}}
$$

The system UGF $\Phi(z)$ after considering the transmission capacity can be derived by applying the following operator $\underset{\varphi}{\otimes}$ over the $\Gamma(z \mid \boldsymbol{L})$ and $\eta(z)$ such that,

$$
\begin{aligned}
\Phi(z \mid \boldsymbol{L}) & =\Gamma(z \mid \boldsymbol{L}) \underset{\varphi}{\otimes} \eta(z) \\
& =\left(\sum_{b}^{B} \pi_{b}(\boldsymbol{L}) \cdot z^{s_{b}(\boldsymbol{L}), d_{b}(\boldsymbol{L})}\right) \underset{\varphi}{\otimes}\left(\sum_{\beta=1}^{Q} \alpha_{\beta} z^{\varsigma_{\beta}}\right) \\
& =\sum_{b}^{B} \sum_{\beta=1}^{Q} \alpha_{\beta} \cdot \pi_{b}(\boldsymbol{L}) \cdot z^{\max \left\{0, d_{b}(\boldsymbol{L})-\min \left(s_{b}(\boldsymbol{L}), \varsigma_{\beta}\right)\right\}} \\
& =\sum_{\theta=1}^{\Theta} \gamma_{\theta}(\boldsymbol{L}) \cdot z^{\hat{d}_{\theta}(\boldsymbol{L})}
\end{aligned}
$$

where $\gamma_{\theta}(\boldsymbol{L})=\mathrm{P}\left\{\hat{D}=\hat{d}_{\theta}(\boldsymbol{L})\right\}$ is for the entire system.

From equation (10), the availability of the system can be computed as follows.

$$
A(\boldsymbol{L}, \boldsymbol{X})=\sum_{\theta=1}^{\Theta} \gamma_{\theta}(\boldsymbol{L}) \cdot I\left(\hat{d}_{\theta}(\boldsymbol{L})=0\right)
$$

where $I(x)$ is an indicator function such that $I(x)=1$ if $x$ is true and $I(x)=0$ if $x$ is not true.

\section{OPTIMIZATION TECHNIQUE}

The optimization model formulated in (15) is a complex combinatorial deterministic optimization problem. It is not realistic to examine all possible solutions exhaustively. In the literature, the genetic algorithm (GA) has been proven efficient in solving large-scale reliability optimization problems $[5,9$, 11, 42, 47-49]. The details of GA can be found in [50]. In this section, the GA is adapted in order to implement the specific optimization problem in this paper.

To solve the optimization problem using GA, the solution must be represented in an integer string. Although the load on each element is generally assumed continuous, we can discretize the load based on the required accuracy. For example, the load $L_{i}$ on element $e_{i}$ can be represented by an integer $k_{i}$ such that,

$$
L_{i}=L_{i, \min }+\frac{k_{i}}{K}\left(L_{i, \max }-L_{i, \min }\right)
$$

where $k_{i}=0,1, \cdots, K$, i.e., the load on element $e_{i}$ can be selected from the $K+1$ possible choices. The value of $K$ determines the required level of accuracy. Another decision variable in the optimization model (15) is the allocation of the 
protection effort $\boldsymbol{X}=\left(x_{1}, \cdots, x_{M}\right)$. Let $\Lambda$ be the maximum protection effort that can be allocated to an element. The protection effort allocated to element $e_{i}$ can be represented by an integer between zero and $\Lambda$, i.e., $x_{i} \in\{0,1, \cdots, \Lambda\}$.

Taking consideration of both loading and protection, we define an integer string $\boldsymbol{Y}=\left(y_{1}, \cdots, y_{M}\right)$ to represent a solution of the optimization model (15), where $y_{i}$ refers to the solution corresponding to element $e_{i}$ and the range of $y_{i}$ is,

$$
0 \leq y_{i}<(K+1)(\Lambda+1)
$$

The integer $y_{i}$ can be decoded into the load and the protection effort in the following manner.

$$
\begin{aligned}
& k_{i}=\left\lceil y_{i} /(\Lambda+1)\right\rceil \\
& x_{i}=\bmod _{(\Lambda+1)}\left(y_{i}\right)
\end{aligned}
$$

where $\lceil x\rceil$ denotes the greatest integer less than $x$ and $\bmod _{x} y=y-\lceil y / x\rceil \cdot x$ denotes the remainder of $y / x$.

For a given $k_{i}$ and $x_{i}$, the corresponding $y_{i}$ is composed as follows.

$$
y_{i}=k_{i} \cdot(\Lambda+1)+x_{i}
$$

After decoding the solutions, the system availability can be computed using the algorithm suggested in Section 5. Given that the resulted system availability is $A$, the fitness value in GA can be evaluated as follows.

$$
\begin{aligned}
C_{G A}= & \sum_{i=1}^{M}\left(c_{p_{1} i} \cdot x_{i}+c_{p_{2} i} \cdot f \cdot v\left(x_{i}, d\right) \cdot T+c_{r i} \cdot \lambda_{i} \cdot e^{\beta_{i} L_{i}} \cdot T\right) \\
& +\omega \cdot I\left(A<A^{*}\right)
\end{aligned}
$$

where $\omega$ is a sufficient large penalty imposed if the pre-specified system availability is not met.

For given parental strings $\boldsymbol{Y}_{1}$ and $\boldsymbol{Y}_{2}$, crossover operator produces offspring string $\boldsymbol{Y}_{3}$ by combining the first $i$ elements of $\boldsymbol{Y}_{1}$ and last $M-i$ elements of $\boldsymbol{Y}_{2}$, where $i$ is an randomly generated integer from 1 to $M$.The mutation operator swaps elements of the string initially located at two randomly chosen positions.

\section{NUMERICAL EXPERIMENTS}

An example of the multi-state system with common bus performance sharing can be found in the collaborative computing system as illustrated in the literature [51]. In this section, we will consider a series-parallel collaborative computing system with 7 multi-state elements and a common bus line for transmitting performance. The structure of the system is shown below in Figure 2. Each element has only one processor which can work at different performance levels depending on its working load. Elements connected in parallel are working together to satisfy the demand of the sub-system. The demand of each sub-system is determined by the complexity of computation problem in the system.

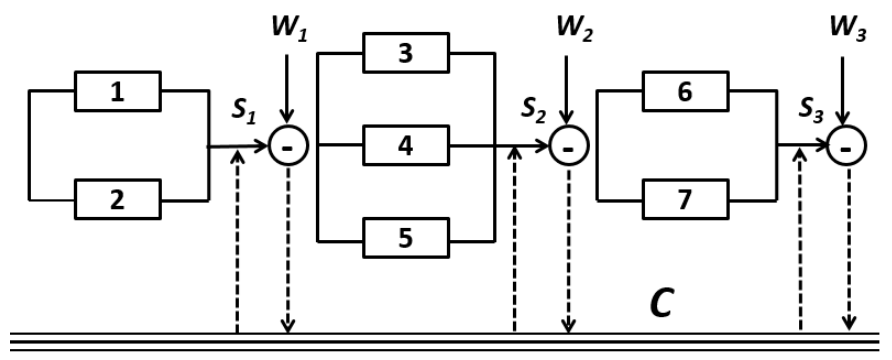

Fig. 2. Series-parallel system with 3 sub-systems and 7 elements

Each element is different and has different working capacities and failure rates. The working capacity can vary in the range $\left[L_{i, \text { min }}, L_{i, \max }\right]$ for each $i=1, \cdots, 7$. The parameters of the elements are summarized in Table 1 . The demand of each sub-system is summarized in Table 2.

TABLE I

PARAMETERS OF MULTI-STATE ELEMENTS

\begin{tabular}{cccccccc}
\hline \hline & $e_{1}$ & $e_{2}$ & $e_{3}$ & $e_{4}$ & $e_{5}$ & $e_{6}$ & $e_{7}$ \\
\hline$\lambda_{i}$ & 0.0105 & 0.0116 & 0.0113 & 0.0109 & 0.0108 & 0.0112 & 0.0095 \\
$\beta_{i}$ & 0.14 & 0.17 & 0.11 & 0.13 & 0.14 & 0.13 & 0.11 \\
$L_{i, \min }$ & 20 & 20 & 30 & 10 & 20 & 30 & 20 \\
$L_{i, \max }$ & 50 & 40 & 60 & 50 & 50 & 50 & 60 \\
$a_{i}$ & 1 & 2 & 2 & 1 & 3 & 2 & 1 \\
$c_{i}$ & 2 & 2 & 2 & 2 & 2 & 2 & 2 \\
$t_{r i}$ & 0.056 & 0.063 & 0.065 & 0.058 & 0.055 & 0.062 & 0.061 \\
$t_{p i}$ & 0.12 & 0.09 & 0.11 & 0.13 & 0.09 & 0.08 & 0.11 \\
$c_{p_{1} i}$ & 5 & 6 & 4 & 3 & 5 & 6 & 7 \\
$c_{p_{2} i}$ & 15 & 16 & 15 & 14 & 16 & 17 & 13 \\
$c_{r i}$ & 15 & 14 & 13 & 12 & 16 & 12 & 14 \\
\hline \hline
\end{tabular}

TABLE II

SUBSYSTEM DEMAND DISTRIBUTION

\begin{tabular}{llllll}
\hline \hline \multicolumn{2}{l}{ Sub-system 1} & \multicolumn{2}{c}{ Sub-system 2} & \multicolumn{2}{c}{ Sub-system 3 } \\
$W$ & $p$ & $W$ & $p$ & $W$ & $p$ \\
\hline 50 & 0.6 & 120 & 0.7 & 80 & 0.5 \\
60 & 0.4 & 140 & 0.3 & 110 & 0.5 \\
\hline \hline
\end{tabular}

In this experiment, the constant frequency of the external impact is assumed to be 0.5 , The expected impact intensity is 30 and contest intensity parameter is 1 , i.e., $f=0.5, d=30$, $m=2$. The system lifetime is assumed to be 60 . Two sets of numerical experiments are conducted. In this first set of 
experiments, the capacity of the performance sharing system is defined to be $C=[600.5 ; 300.5]$, and the capacity is $C=[200.5 ; 100.5]$ for the second set of experiments. The protection investment on each element is assumed to be from zero to 50 .

TABLE III

OPTIMAL LOADING AND PROTECTION WITH HIGH TRANSMISSION CAPACITY

\begin{tabular}{|c|c|c|c|c|c|c|c|}
\hline & $e_{1}$ & $e_{2}$ & $e_{3}$ & $e_{4}$ & $e_{5}$ & $e_{6}$ & $e_{7}$ \\
\hline \multicolumn{8}{|c|}{$A^{*}=0.85, A=0.8500, C_{T}=3928, C_{\text {rep }}=2476, C_{i n v}=1452$} \\
\hline$L$ & 20 & 20 & 30 & 10 & 20 & 30 & 20 \\
\hline$X$ & 47 & 37 & 47 & 47 & 37 & 37 & 37 \\
\hline \multicolumn{8}{|c|}{$A^{*}=0.90, A=0.9000, C_{T}=4363, C_{\text {rep }}=3409, C_{i n v}=954$} \\
\hline$L$ & 20 & 20 & 33 & 18 & 20 & 30 & 28 \\
\hline$X$ & 22 & 22 & 35 & 33 & 22 & 22 & 33 \\
\hline \multicolumn{8}{|c|}{$A^{*}=0.92, A=0.9201, C_{T}=4671, C_{r e p}=3924, C_{i n v}=747$} \\
\hline$L$ & 20 & 20 & 30 & 22 & 26 & 30 & 28 \\
\hline$X$ & 22 & 22 & 22 & 23 & 18 & 22 & 18 \\
\hline \multicolumn{8}{|c|}{$A^{*}=0.95, A=0.9504, C_{T}=6125, C_{r e p}=5423, C_{i n v}=702$} \\
\hline$L$ & 23 & 24 & 36 & 30 & 26 & 32 & 40 \\
\hline$X$ & 2 & 36 & 36 & 14 & 36 & 2 & 14 \\
\hline
\end{tabular}

TABLE IV

OPTIMAL LOADING AND PROTECTION WITH LOW TRANSMISSION CAPACITY

\begin{tabular}{|c|c|c|c|c|c|c|c|}
\hline & $e_{1}$ & $e_{2}$ & $e_{3}$ & $e_{4}$ & $e_{5}$ & $e_{6}$ & $e_{7}$ \\
\hline \multicolumn{8}{|c|}{$A^{*}=0.85, A=0.8501, C_{T}=4925, C_{r e p}=3677, C_{i n v}=1248$} \\
\hline$L$ & 20 & 22 & 33 & 30 & 26 & 32 & 20 \\
\hline$X$ & 35 & 34 & 34 & 23 & 43 & 34 & 35 \\
\hline \multicolumn{8}{|c|}{$A^{*}=0.88, A=0.8803, C_{T}=5371, C_{r e p}=4003, C_{i n v}=1368$} \\
\hline$L$ & 26 & 24 & 36 & 30 & 26 & 30 & 28 \\
\hline$X$ & 26 & 44 & 44 & 34 & 44 & 49 & 26 \\
\hline \multicolumn{8}{|c|}{$A^{*}=0.90, A=0.9001, C_{T}=6786, C_{r e p}=5296, C_{i n v}=1490$} \\
\hline$L$ & 29 & 24 & 39 & 30 & 29 & 36 & 36 \\
\hline$X$ & 46 & 33 & 46 & 12 & 46 & 46 & 48 \\
\hline \multicolumn{8}{|c|}{$A^{*}=0.92, A=0.9201, C_{T}=7096, C_{r e p}=5569, C_{i n v}=1527$} \\
\hline$L$ & 29 & 26 & 39 & 38 & 29 & 30 & 36 \\
\hline$X$ & 41 & 42 & 42 & 44 & 44 & 45 & 40 \\
\hline
\end{tabular}

The numerical results are summarized in Table 3 and Table 4 for high transmission capacity and low transmission capacity respectively. They are obtained by running GA for 1000 iterations. In the tables, $A^{*}$ refers to the pre-specified system availability requirement and $A$ is the actual system availability achieved. $C_{T}$ denotes the total system cost. $C_{r e p}$ denotes all repair costs which include both repair cost of internal failures and repair cost caused by external impacts. $C_{i n v}$ denotes the investment on protection.Consistent with our intuition, the results indicate that the total system cost increases with more stringent system availability requirement. Higher transmission capacity reduces the system cost given the same system availability requirement. However, the optimal trade-off between repair cost and protection investment can be different under different settings. In general, no trend can be found if we observe the repair cost and protection cost separately.

It is also interesting to test how the variation of the impact intensity $d$ and the frequency $f$ affect the system cost. To analyze the effect of $d$, we use the parameter values in Table 1 and 2.The low transmission capacity for the performance sharing system is assumed and the pre-specified system availability requirement is set to be 0.85 . By varying $d$ from 10 to 100 , the minimal system cost is found by running GA when the frequency is kept to be 0.5. The results are shown in Table 5. Similarly, the minimal system cost is found for different values of frequency while the impact intensity is kept to be 30 as shown in Table 6.

TABLE V EFFECT OF IMPACT INTENSITY

\begin{tabular}{cccccc}
\hline \hline$d$ & 10 & 20 & 30 & 40 & 50 \\
$C_{T}$ & 3283 & 4047 & 4925 & 5344 & 6065 \\
\hline$d$ & 60 & 70 & 80 & 90 & 100 \\
$C_{T}$ & 6568 & 7010 & 7585 & 7956 & 8456 \\
\hline \hline
\end{tabular}

TABLE VI

EFFECT OF FREQUENCY

\begin{tabular}{cccccc}
\hline \hline$f$ & 0.1 & 0.2 & 0.3 & 0.4 & 0.5 \\
$C_{T}$ & 2689 & 3680 & 4038 & 4346 & 4925 \\
\hline$f$ & 0.6 & 0.7 & 0.8 & 0.9 & 1 \\
$C_{T}$ & 5362 & 5857 & 6058 & 6155 & 6428 \\
\hline \hline
\end{tabular}

The numerical results in both tables clearly indicate that the system cost is an increasing function of $f$ and $d$. The results are consistent with the contest function model as well as the system cost function in (15). The sensitivity analysis of $d$ and $f$ implies that reducing the potential impact frequency and the expect intensity can save the future system operation cost when designing an engineering system.

\section{CONCLUSION}

In this paper, we integrate the effect of loading on element failure rate and the protection of system element against external impacts into a multi-state series-parallel system with common bus performance sharing. Different from previous studies, the performance distribution of each multi-state element is unknown. The performance of each element depends on the load it is carrying. In addition, protection effort is able to reduce the system failure caused by external impacts such as nature disasters and terrorism. Although higher load on each element results in higher performance, the element also tends to fail more frequently. Hence, the repair cost increases and element availability reduces. This paper formulates an 
optimization model to balance the repair cost and protection investment. An availability evaluation algorithm based on universal generation function is suggested for the proposed system. A collaborative computing system is analyzed as an example to illustrate the proposed model and suggest algorithms.

\section{REFERENCES}

[1] G.Levitin, "Multi-state series-parallel system expansion-scheduling subject to availability constraints," IEEE Trans. Reliab., vol. 49, no.1, pp.71-79, Mar. 2000.

[2] Z. Wang, K. Tang and X. Yao, "A memetic algorithm for multi-level redundancy allocation,"IEEE Trans. Reliab., vol. 59, no. 4, pp.754-765, Dec. 2010.

[3] V. K.Sharma, M.Agarwal and K.Sen, "Reliability evaluation and optimal design in heterogeneous multi-state series-parallel systems," Inf. Sci., vol. 181, no. 2, pp. 362-378, 2011.

[4] Y. F. Li and R. Peng, "Availability modeling and optimization of dynamic multi-state series-parallel systems with random reconfiguration,"Reliab. Eng. Syst. Saf., vol. 127,pp. 47-57, 2014.

[5] Z. Tian, G. Levitin and M. J. Zuo, "A joint reliability-redundancy optimization approach for multi-state series-parallel systems,"Reliab. Eng. Syst. Saf., vol. 94, no. 10, pp. 1568-1576, 2009.

[6] C. D. Dao, M. J. Zuo and M. Pandey, "Selective maintenance for multi-state series-parallel systems under economic dependence,"Reliab. Eng. Syst. Saf., vol. 121, pp. 240-249, 2014.

[7] A. Lisnianski and Y. Ding, "Redundancy analysis for repairable multi-state system by using combined stochastic processes methods and universal generating function technique,"Reliab. Eng. Syst. Saf., vol. 94, no. 11, pp. 1788-1795, 2009.

[8] G. Levitin, "Reliability of multi-state systems with common bus performance sharing,"IIE Trans., vol. 43, pp.518-524, 2011.

[9] H. Xiao and R. Peng, "Optimal allocation and maintenance of multi-state elements in series-parallel systems with common bus performance,"Comput. Ind. Eng., vol. 72, pp. 143-151, 2014.

[10] Y. Zhou, Z. Zhang, T. R. Lin and L. Ma, "Maintenance optimization of a multi-state series-parallel system considering economic dependence and state-dependent inspection intervals,"Reliab. Eng. Syst. Saf., vol. 111, pp. 248-259, 2013.

[11] M. A. Ardakan and A. Z. Hamadani, "Reliability optimization of series-parallel systems with mixed redundancy strategy in subsystems,"Reliab. Eng. Syst. Saf., vol. 130, pp.132-139, 2014.

[12] R. K. Iyer and D. J. Rossetti, "A measurement-based model for workload dependence of CPU errors,"IEEE Trans. Comput., vol. 35, no. 6, pp. 511-519, Jun. 1986.

[13] K. C. Kapur and L. R. Lamberson, Reliability in engineering design. New York: Wiley, 1977.

[14] G. Levitinamd S. Amari, "Optimal load distribution in series-parallel systems,"Reliab. Eng. Syst. Saf., vol. 94. no. 2, pp. 254-260, 2009.

[15] H. Xiao, R. Peng, W. Wang and F. Zhao, "Optimal element loading for linear sliding window systems," Proc. Inst. Mech. Eng. O: J. Risk Reliab., doi:10.1177/1748006X15580218, 2015.

[16] G. Levitin, L. Xing and Y. Dai, "Optimal loading of system with random repair time,"Eur. J. Oper. Res., vol.247, no.1, pp. 137-143, 2015.

[17] G. Levitin, L. Xing and Y. Dai, "Optimal completed work dependent loading of components in cold standby systems," Int. J. Gen. Syst.,vol. 44, no. 4, pp. 471-484, 2015.

[18] G. Levitin, L. Xing and Y. Dai, "Optimal component loading in 1-out-of-N cold standby systems,"Reliab. Eng. Syst. Saf., vol.127, pp. 58-64, 2014.

[19] M. Nourelfath and F. Yalaoui, "Integrated load distribution and production planning in series-parallel multi-state systems with failure rate depending on load,"Reliab. Eng. Syst. Saf., vol. 106, pp. 38-145, 2012.

[20] J. Zhuang and V. M. Bier, "Balancing terrorism and natural disasters-defensive strategy with endogenous attacker effort,"Oper. Res. vol. 55, no. 5, pp. 976-991, 2007.

[21] M. N. Azaiez and V. M. Bier, "Optimal resource allocation for security in reliability systems,"Eur. J. Oper. Res., vol. 181, no.2, pp. 773-786, 2007.

[22] G. Brown, M. Carlyle, J. Salmer' on and K. Wood, "Defending critical infrastructure," Interfaces, vol. 36, no. 6, pp. 530-544, 2006.
[23] M. Golalikhani and J. Zhuang "Modeling arbitrary layers of continuous level defenses in facing with a strategic attacker,"Risk Anal., vol. 31, no.4, pp. 533-547, 2011.

[24] G. Levitin and K. Hausken, "Is it wise to protect false targets?" Reliab. Eng. Syst. Saf., vol. 96. no. 2, pp. 1647-1656, 2011.

[25] R. Peng, G. Levitin, M. Xie and S. H. Ng, "Optimal defence of single object with imperfect false target,"J. Oper. Res. Soc., vol. 62, pp. 134-141, 2011.

[26] G. Levitin, K. Hausken and Y. S. Dai, "Individual vs. overarching protection for minimizing the expected damage caused by an attack,"Reliab. Eng. Syst. Saf., vol. 119, pp. 117-125, 2013.

[27] R. Peng, L. Guo, G. Levitin, H. D. Mo and W. B. Wang, "Maintenance versus individual and overarching protections for parallel systems," Qual. Technol. Quant. Manag., vol. 11, no.3, pp. 353-362, 2014.

[28] L. A. Escobar and W. Q. Meeker, "A review of accelerated test models," Statistical Science," vol. 21, no.4, pp. 552-577, 2006.

[29] D. R. Cox, "Regression models and life tables (with discussion)," J. R. Stat. Soc. Ser. B-Stat. Methodol., vol. 34, pp. 187-220, 1972.

[30] I. J. Jozwiak, "An introduction to the studies of reliability of systems using the Weibull proportional hazards model,"Microelectron. Reliab., vol. 37, no. 6, pp. 915-918, 1997.

[31] Z. G. Li, S. Y. Zhou, C. Sievenpiper and S. Choubey, "Change detection in the cox proportional hazards models from different reliability data,"Qual. Reliab. Eng. Int., vol. 26, no. 7, pp. 677-689, 2010.

[32] A. Tiwari and D. Roy, "Estimation of reliability of mobile handsets using Cox-proportional hazard model,"Microelectron. Reliab., vol. 53, no.3, pp. 481-487, Mar. 2013.

[33] S. Skaperdas, "Contest success functions,"Econ. Theory, vol. 7, pp. 283-290, 1996.

[34] K. Hausken, Production and conflict models versus rent seeking models. Public Choice, vol. 123, pp. 59-93, 2005.

[35] I. Ushakov, "Universal generating function,"Sov. J. Comput. Syst. Sci., vol. 24, pp. 118-129, 1986.

[36] W. C. Yeh, "A convolution universal generating function method for evaluating the symbolic one-to-all-target-subset reliability functions of acyclic multi-state information networks." IEEE Trans. Reliab., vol. 58, no. 3, pp. 476-484, Sep. 2009.

[37] Y. Ding, M. J. Zuo and A. Lisnianski, "A framework for reliability approximation of multi-State weighted k-out-of-n systems," IEEE Trans. Reliab., vol. 59, no. 2, pp. 297-308, Jun. 2010.

[38] G.Levitin, "Reliability of linear multistate multiple sliding window systems,"Nav. Res. Logist., vol. 52, no., 3, pp. 212-223, Mar. 2005.

[39] G.Levitin, "Linear multi-state sliding-window systems," IEEE Trans. Reliab., vol. 52, no. 2, pp. 263-269, Jun. 2003.

[40] G.Levitin and Y. S. Dai, "k-out-of-n sliding window systems,"IEEE Trans. Syst. Man Cybern. Paart A-Syst. Hum., vol. 42, no. 3, pp. 707-714 May. 2012.

[41] G.Levitin, "Threshold optimization for weighted voting classifiers," Nav. Res. Logist.,vol. 50, no. 4, pp. 322-344, Jun. 2003.

[42] R. Peng, M. Xie, S. H. Ng and G. Levitin, "Element maintenance and allocation for linear consecutively connected systems,"IIE Trans., vol. 44, no. 11, pp. 964-973, 2012.

[43] Y. F. Li and E. Zio, "A multi-state model for the reliability assessment of a distributed generation system via universal generating function," Reliab. Eng. Syst. Saf., vol.106, pp. 28-36, 2012.

[44] Z. W. An, H. Z. Huang and Y. Liu, "A discrete stress-strength interference model based on universal generating function,"Reliab. Eng. Syst. Saf., vol. 93, no. 10, pp. 1485-1490, 2008.

[45] S. Faghih-Roohi, M. Xie, K.M Ng and R. C. M. Yam, "Dynamic availability assessment and optimal component design of multi-state weighted k-out-of-n systems,"Reliab. Eng. Syst. Saf., vol. 123, pp. 57-62, 2014.

[46] G. Levitin, The universal generating function in reliability analysis and optimization. London :Springer-Verlag, 2005.

[47] Y. Ding, P. Wang and A. Lisnianski, "Optimal reserve management for restructured power generating systems,"Reliab. Eng. Syst. Saf., vol. 91, no. 7, pp. 792-799, 2006.

[48] Y. K. Lin and C. T. Yeh, "Maximal network reliability with optimal transmission line assignment for stochastic electric power networks via genetic algorithms,"Appl. Soft. Comput., vol. 11, no. 2, pp. 2714-2724, 2011.

[49] A. Lisnianski, I. Frenkel and Y. Ding, Multi-state system reliability analysis and optimization for engineers and industrial managers, Springer-Verlag, London, Heidelberg, New York, 2010. 
[50] J. H. Holland, Adaptation in natural and artificial systems, Ann Arbor: University of Michigan Press, (1975.

[51] H. Yu, J. Yang and H. D. Mo, "Reliability analysis of repairable multi-state system with common bus performance sharing," Reliab. Eng. Syst. Saf., vol. 132, pp. 90-96, 2014. 